\title{
Detailed study of the superconducting properties in compressed germane
}

\author{
Radosław Szczȩśniak and Artur P. Durajskia \\ Institute of Physics, Częstochowa University of Technology, Ave. Armii Krajowej 19, 42-200 Czȩstochowa, Poland
}

Received 18 September 2015 / Received in final form 28 October 2015

Published online 21 December 2015

(C) The Author(s) 2015. This article is published with open access at Springerlink.com

\begin{abstract}
Hydrogen-rich compounds under extreme pressure are the most promising systems for searching a high-temperature superconductivity. In presented paper, we report analysis of the thermodynamic properties of hydrogenated germanium (germane, $\mathrm{GeH}_{4}$ ) at $220 \mathrm{GPa}$ obtained within the framework of the Migdal-Eliashberg theory. We observe that together with the increase of Coulomb pseudopotential from 0.1 to 0.3 the critical temperature decreases from $92.36 \mathrm{~K}$ to $52.80 \mathrm{~K}$. A similar trend is also well-visible in the case of other thermodynamic properties. Moreover, we study the influence of external pressure on the superconducting state of $\mathrm{GeH}_{4}$. On this basis we conclude that increase of pressure from 20 to $220 \mathrm{GPa}$ has a pronounced effect on the thermodynamic stability of germane. Finally, it is proved that the properties of the superconducting state of $\mathrm{GeH}_{4}$ differ markedly from predictions of the Bardeen-Cooper-Schrieffer (BCS) theory.
\end{abstract}

\section{Introduction}

The possibility of high-temperature superconductivity in simple elements enriched with hydrogen was theoretically predicted by Ashcroft in 2004 [1]. It was suggested that due to the chemical precompression of hydrogen caused by atoms of heavier elements, the hydrogen-rich compounds can become metallic and superconducting at considerably lower external pressure than may be necessary for pure hydrogen [2]. The exploration of potential superconductivity in these compounds is thus an obvious trend. A numerous theoretical and experimental studies have been performed in the last few years [3]. From the experimental point of view, the superconductivity was found in silane $\left(\mathrm{SiH}_{4}\right)$ [4], hydrogen sulfide $\left(\mathrm{H}_{3} \mathrm{~S}\right)$ [5] and hydride phosphine $\left(\mathrm{PH}_{3}\right)$ [6] at high pressures. Wherein the measured critical temperature $\left(T_{C}\right)$ of $203 \mathrm{~K}$ in hydrogen sulfide is among the highest over allknown superconductors [5]. The theoretical studies have revealed a significantly more superconducting hydrogenrich materials such as $\mathrm{PtH}[7,8], \mathrm{H}_{2} \mathrm{~S}$ [9], $\mathrm{GaH}_{3}$ [10,11], $\mathrm{ScH}_{3}$ [12,13], $\mathrm{SnH}_{4}$ [14], $\mathrm{GeH}_{4}[15,16], \mathrm{NbH}_{4}$ [17,18], $\mathrm{CaH}_{6}$ [19], $\mathrm{MgH}_{6}$ [20] of which the highest critical temperature equal to $263-271 \mathrm{~K}$ was estimated for $\mathrm{MgH}_{6}$ compound at a pressure range from 300 to $400 \mathrm{GPa}$ [20]. As it turns out pressure $(p)$ can have a very large impact on the critical temperature and thermodynamics of superconducting state. From technological point of view,

\footnotetext{
${ }^{a}$ e-mail: adurajski@wip.pcz.pl
}

to study the behaviour of materials as a function of very high pressure it is necessary to use a diamond-anvil cell in which it is possible to achieve static pressures about $260 \mathrm{GPa}$. In the case of first-principles calculations there are no limits and, for example, the atomic metallic hydrogen has been studied up to a pressure of $3500 \mathrm{GPa}$ [21].

In the present paper, we report the systematic and detailed study of superconducting state in germane $\left(\mathrm{GeH}_{4}\right)$ at $220 \mathrm{GPa}$ for a stable structure $C 2 / \mathrm{c}$. Germane, together with silane and stannane, belongs to the group IVa hydrides. Whilst the several experimental measurements, have been performed to investigate the superconducting state induced in $\mathrm{SiH}_{4}[4,22,23]$, no study on the hydrides of heavier group IV elements such as $\mathrm{GeH}_{4}$ and $\mathrm{SnH}_{4}$ has been attempted. So far, the theoretical studies have predicted high superconductivities with $T_{C}$ reaching $64 \mathrm{~K}$ at $220 \mathrm{GPa}$ for $\mathrm{GeH}_{4}$ [15] and $62 \mathrm{~K}$ at $200 \mathrm{GPa}$ for $\mathrm{SnH}_{4}$ [24] (for comparison: $T_{C}=17 \mathrm{~K}$ for $\mathrm{SiH}_{4}$ at $220 \mathrm{GPa}$ [25]). Moreover, some estimates show that since the atomic radius and atomic masses of $\mathrm{Ge}$ and $\mathrm{Sn}$ are larger than $\mathrm{Si}$, $\mathrm{GeH}_{4}$ and $\mathrm{SnH}_{4}$ might be easier to become a metal (at a lower metallization pressure) than silane [26].

The paper is organized as follows. Next section contains a short outline of the strong-coupling Eliashberg formalism. In Section 3, we present the thermodynamic properties of superconducting $\mathrm{GeH}_{4}$ at $220 \mathrm{GPa}$ and we discuss the influence of pressure on the superconducting state of $\mathrm{GeH}_{4}$ by comparison of results obtained here with the results reported previously for $\mathrm{GeH}_{4}$ at $20 \mathrm{GPa}(\mathrm{Cmmm}$ phase) [27]. Section 4 summarizes the obtained results. 
A
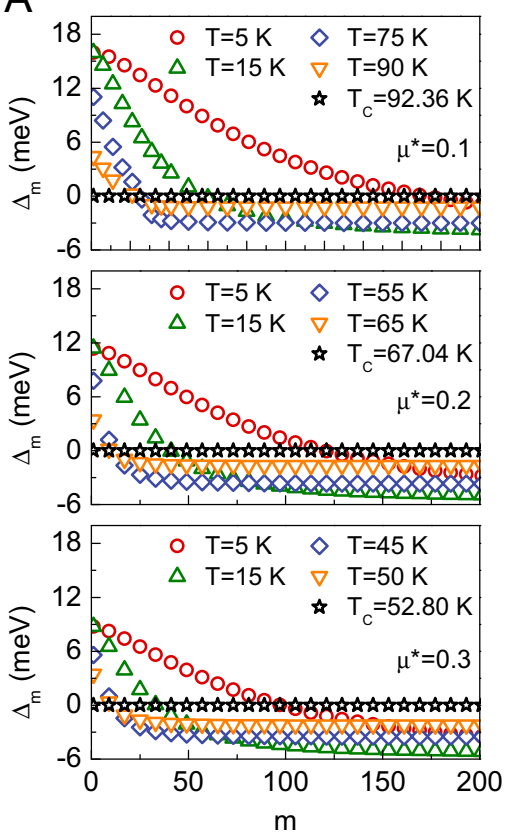

B

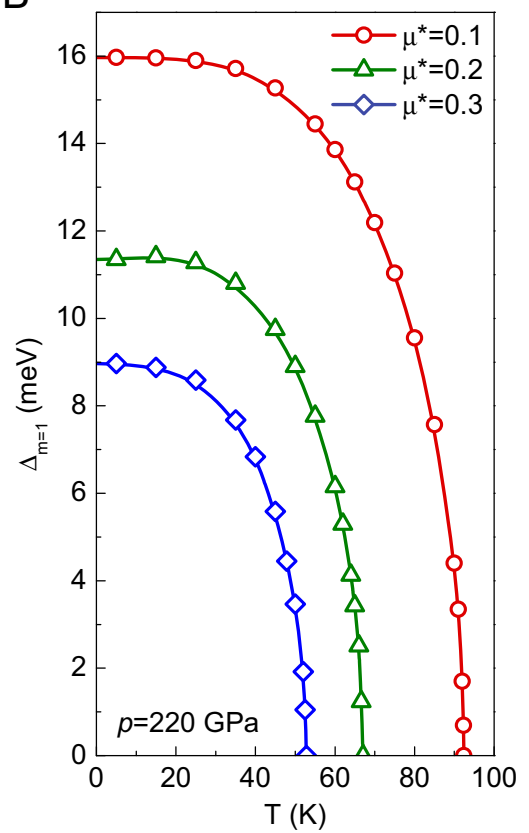

C

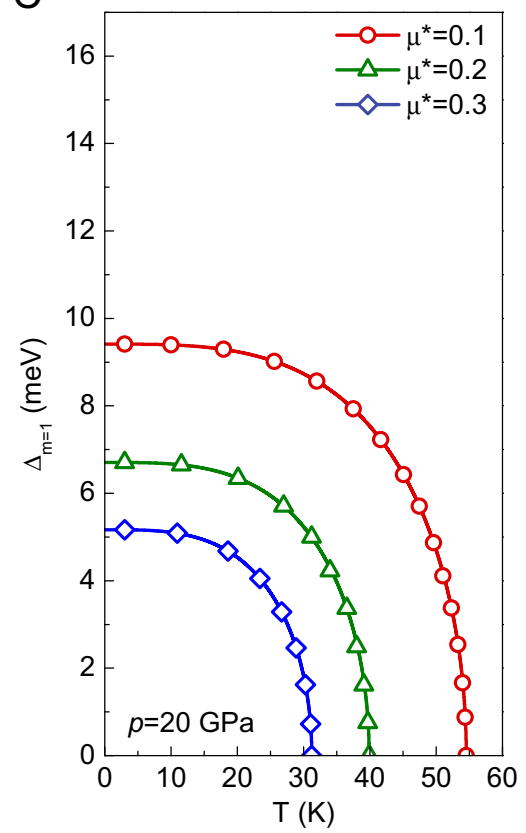

Fig. 1. (A) The form of the order parameter on the imaginary axis for the selected values of the temperature and the Coulomb pseudopotential $(p=220 \mathrm{GPa})$. The full shape of the order parameter for $m=1$ as the function of temperature for the selected values of the Coulomb pseudopotential for (B) $p=220 \mathrm{GPa}$ and (C) $p=20 \mathrm{GPa}$ [27].

\section{Eliashberg formalism}

Our investigations are conducted within the framework of the strong-coupling Migdal-Eliashberg theory of superconductivity [28], which accurately treats the electron-phonon interaction and offers a way to exact calculate the critical temperature, superconducting energy gap, specific heat and thermodynamic critical field. The Eliashberg equations in the single-band case formulated on the imaginary frequency axis take the following form [28,29]:

$$
\phi_{n}=\frac{\pi}{\beta} \sum_{m=-M}^{m=M} \frac{\lambda\left(i \omega_{n}-i \omega_{m}\right)-\mu^{\star} \theta\left(\omega_{c}-\left|\omega_{m}\right|\right)}{\sqrt{\omega_{m}^{2} Z_{m}^{2}+\phi_{m}^{2}}} \phi_{m}
$$

and

$$
Z_{n}=1+\frac{1}{\omega_{n}} \frac{\pi}{\beta} \sum_{m=-M}^{m=M} \frac{\lambda\left(i \omega_{n}-i \omega_{m}\right)}{\sqrt{\omega_{m}^{2} Z_{m}^{2}+\phi_{m}^{2}}} \omega_{m} Z_{m}
$$

where $\phi_{n}=\Delta_{n} Z_{n}, \Delta_{n} \equiv \Delta\left(i \omega_{n}\right)$ denotes the superconducting order parameter and $Z_{n} \equiv Z\left(i \omega_{n}\right)$ is the wave function renormalization factor. Moreover, the quantity $\theta$ denotes the Heaviside function, $\omega_{n}$ are the Matsubara frequencies defined in the following way: $\omega_{n} \equiv$ $(\pi / \beta)(2 n-1)$, where $n=0, \pm 1, \pm 2, \ldots, \pm M$, and $M=$ 1100. Symbol $\beta$ denotes an inversion of temperature $\beta \equiv$ $\left(k_{B} T\right)^{-1}$ and $\mu^{\star}$ represents the Coulomb pseudopotential with a cut-off frequency $\omega_{c}$ equals three times the maximum phonon frequency $\left(\omega_{c}=3 \Omega_{\max }\right.$, where $\Omega_{\max }=$ $331 \mathrm{meV}$ [15]). In the Eliashberg equations, the Coulomb pseudopotential is treated as a parameter that should be fitted in order to reproduce the experimental value of critical temperature. In the case of $\mathrm{GeH}_{4}$ at $220 \mathrm{GPa}$, the absence of experimental data caused that we conducted our calculations for a wide range of $\mu^{\star}$, from 0.1 to 0.3 . Furthermore, in equations (1) and (2), $\lambda\left(i \omega_{n}-i \omega_{m}\right)$ is a pairing kernel for the electron-phonon interaction:

$$
\lambda\left(i \omega_{n}-i \omega_{m}\right) \equiv 2 \int_{0}^{\Omega_{\max }} d \Omega \frac{\Omega}{\left(\omega_{n}-\omega_{m}\right)^{2}+\Omega^{2}} \alpha^{2} F(\Omega) .
$$

The central quantity of the Migdal-Eliashberg theory is the Eliashberg spectral function $\alpha^{2} F(\Omega)$, which expresses the electron-phonon interaction. For $\mathrm{GeH}_{4}$ at $220 \mathrm{GPa}$ (metallic monoclinic structure of $C 2 / c$ ) the $\alpha^{2} F(\Omega)$ function was determined in paper [15] from the ab-initio calculations (Quantum-ESPRESSO package [30]) and was used as a input element into the Eliashberg equations to determine, inter alia, $T_{C}$ and the temperature dependence of the energy gap. For this purpose, in the Eliashberg equations for a fixed value of $\mu^{\star}$ we have increased the value of temperature until we have reached the equality $\Delta_{m=1}=0$ at $T=T_{C}$.

\section{Results and discussion}

The dependence of the order parameter on the successive Matsubara frequencies for selected values of $\mu^{\star}$ and temperature is presented in Figure 1A. It can be noticed, that with the growth of temperature the maximum of the order parameter function $\left(\Delta_{m=1}\right)$ is decreasing. The full dependence of $\Delta_{m=1}(T)$ are presented in 

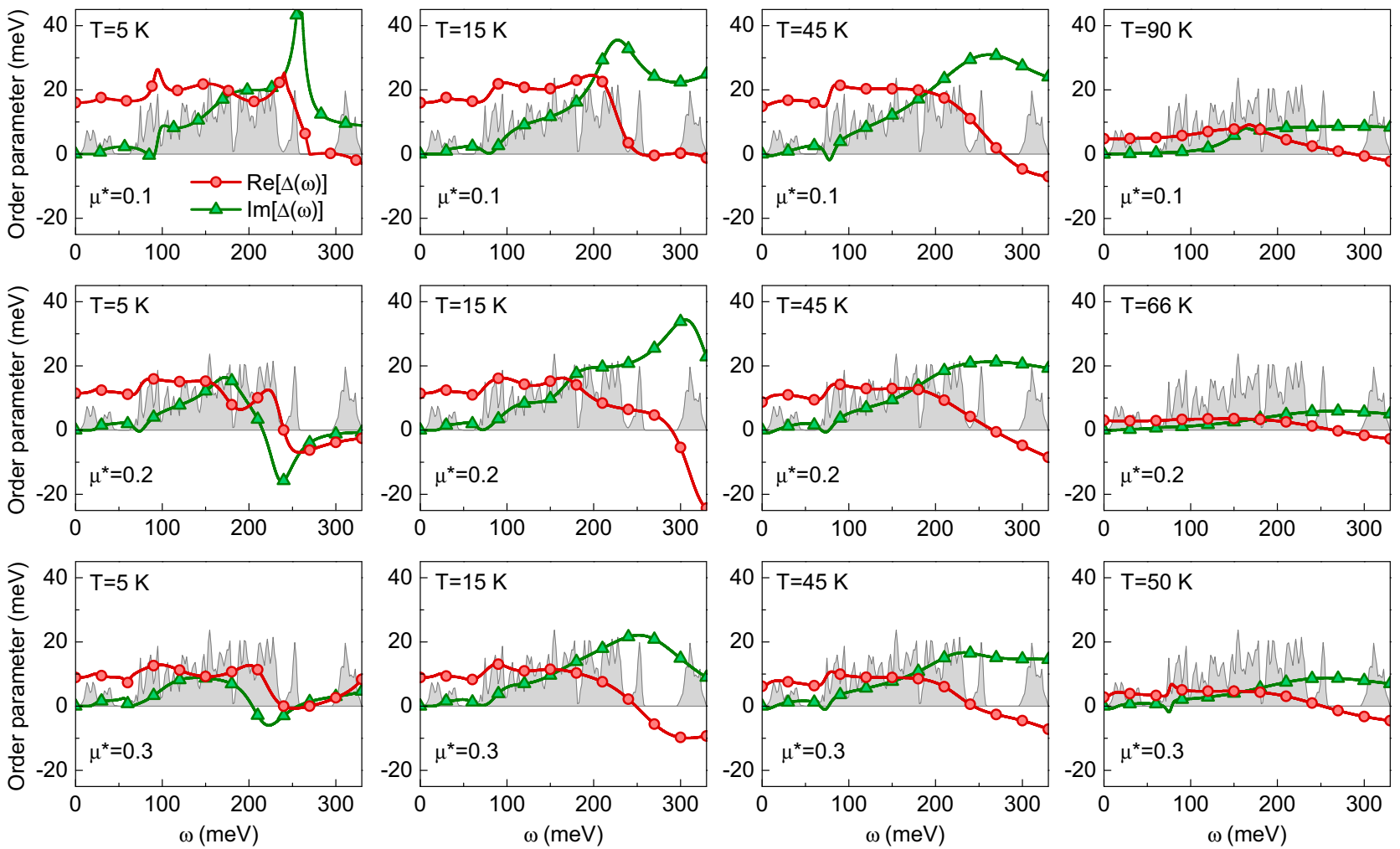

Fig. 2. The real and imaginary part of the order parameter on the real axis at $220 \mathrm{GPa}$ for selected values of the temperature and the Coulomb pseudopotential. Additionally, the rescaled Eliashberg function has been plotted $\left(40 \alpha^{2} F(\Omega)\right)$.

Table 1. Influence of Coulomb pseudopotential on the value of critical temperature and zeroth-temperature energy gap at the Fermi level for the hydrogenated germanium at $20 \mathrm{GPa}[27]$ and $220 \mathrm{GPa}$.

\begin{tabular}{ccccccc}
\hline & \multicolumn{3}{c}{$T_{C}(\mathrm{~K})$} & \multicolumn{3}{c}{$\Delta(0)(\mathrm{meV})$} \\
\hline$\mu^{\star}$ & 0.1 & 0.2 & 0.3 & 0.1 & 0.2 & 0.3 \\
\hline $\mathrm{GeH}_{4} 20 \mathrm{GPa}$ & 54.54 & 39.89 & 31.23 & 9.57 & 6.79 & 5.22 \\
$\mathrm{GeH}_{4} 220 \mathrm{GPa}$ & 92.36 & 67.04 & 52.80 & 16.29 & 11.54 & 8.89 \\
\hline
\end{tabular}

Figure 1B. On this basis we can notice that, for the investigated systems, critical temperature decreases from 92.36 K to $52.80 \mathrm{~K}$ when Coulomb pseudopotential increases from 0.1 to 0.3 . At this point it should be noted that in the strong coupling systems the Allen-Dynes modified McMillan equation gives underestimate value of $T_{C}$ (64 K for $\left.\mu^{\star}=0.13[15]\right)$.

Our results for $\mathrm{GeH}_{4}$ at $220 \mathrm{GPa}$ (Figs. 1A and 1B) are supplemented with the results obtained previously for $\mathrm{GeH}_{4}$ at $20 \mathrm{GPa}$ (Fig. 1C) [27]. More precisely the effect of pressure on the superconducting state in $\mathrm{GeH}_{4}$ compound can be traced by analysing the results presented in Table 1 , where $\Delta(0)$ denotes the physical value of energy gap. The energy gap was determined on the basis of the results from imaginary axis solutions used as a input data to the Eliashberg equations defined in the mixed representation (both on the real and imaginary frequency axis) $[29,31]$.
In particular based on the form of the order parameter on the real axis, in the Eliashberg formalism, the exact values of the superconducting energy gap can be obtained using the following equation [29]:

$$
\Delta(T)=\operatorname{Re}[\Delta(\omega=\Delta(T))] .
$$

The results of $\Delta(\omega)$, for selected values of temperature and for fixed values of the Coulomb pseudopotential are presented in Figure 2. We can see that for the low frequencies, the zero value is taken only by the imaginary part of $\Delta(\omega)$. This proves that in the considered range of frequencies the damping effects not exist. In the case of real part we can observe a clear correlation with the shape of the Eliashberg spectral function, which is plotted in the background of Figure 2.

In the next step, we calculated the thermodynamic critical field:

$$
\frac{H_{C}}{\sqrt{\rho(0)}}=\sqrt{-8 \pi[\Delta F / \rho(0)]}
$$

where symbol $\Delta F=F^{S}-F^{N}$ denotes the free energy difference between the superconducting and the normal state [32]:

$$
\begin{aligned}
\frac{\Delta F}{\rho(0)}= & -\frac{2 \pi}{\beta} \sum_{n=1}^{M}\left(\sqrt{\omega_{n}^{2}+\Delta_{n}^{2}}-\left|\omega_{n}\right|\right) \\
& \times\left(Z_{n}^{S}-Z_{n}^{N} \frac{\left|\omega_{n}\right|}{\sqrt{\omega_{n}^{2}+\Delta_{n}^{2}}}\right) .
\end{aligned}
$$



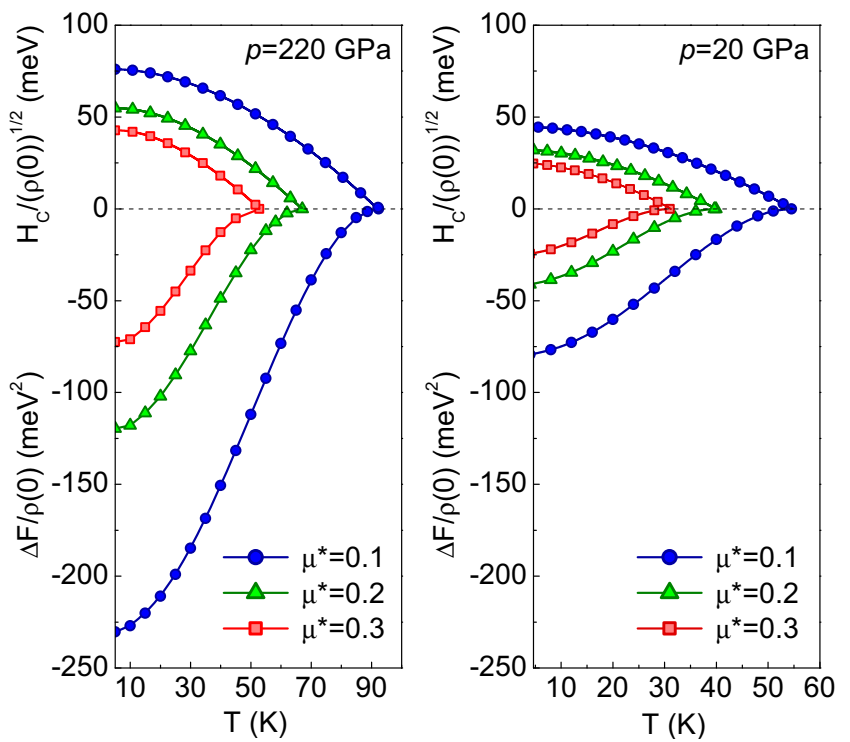

Fig. 3. The thermodynamic critical field and the free energy difference between the superconducting and normal state as a function of temperature for selected values of the Coulomb pseudopotential. The results for $\mathrm{GeH}_{4}$ at $20 \mathrm{GPa}$ are adopted from paper [27].

Here, $\rho(0)$ is the electron density of states at the Fermi level and symbols $Z_{n}^{S}$ and $Z_{n}^{N}$ are the mass renormalization functions for the superconducting and for the normal state, respectively. Let us note that equation (6) can be computed on the basis of the solutions of the imaginary axis Eliashberg equations.

The temperature dependence of thermodynamic critical field and free energy difference is presented in Figure 3 . We can observe a large variations between results obtained for a sample at $20 \mathrm{GPa}$ and $220 \mathrm{GPa}$. From the physical point of view, the thermodynamically more stable is system at $220 \mathrm{GPa}$, because in this case the absolute values of $\Delta F$ are larger than in the second case for the corresponding values of $\mu^{\star}$.

Also on the basis of the free energy difference, we calculated the specific heat difference between the superconducting and normal states $\left(\Delta C=C^{S}-C^{N}\right)$ :

$$
\frac{\Delta C}{k_{B} \rho(0)}=-\frac{1}{\beta} \frac{d^{2}[\Delta F / \rho(0)]}{d\left(k_{B} T\right)^{2}} .
$$

Let us note that the specific heat for the normal state is defined as: $C^{N}=\gamma T$, where symbol $\gamma$ denotes the Sommerfeld constant: $\gamma \equiv(2 / 3) \pi^{2} k_{B}^{2} \rho(0)(1+\lambda)$. In Figure 4 we have results obtained for $\mathrm{GeH}_{4}$ at $220 \mathrm{GPa}$ and compared with those computed for $\mathrm{GeH}_{4}$ at $20 \mathrm{GPa}$ [27].

One of the most convenient way to compare superconducting systems is to determine dimensionless ratios connected with thermodynamic magnitudes: $2 \Delta(0) / k_{B} T_{C}$, $\Delta C\left(T_{C}\right) / C^{N}\left(T_{C}\right)$ and $T_{C} C^{N}\left(T_{C}\right) / H_{C}^{2}(0)$. In accordance with the BCS theory, the above ratios take the universal values: $3.53,1.43,0.168$, respectively $[33,34]$. However, due to the strong-coupling and retardation effects taken into account in the Migdal-Eliashberg
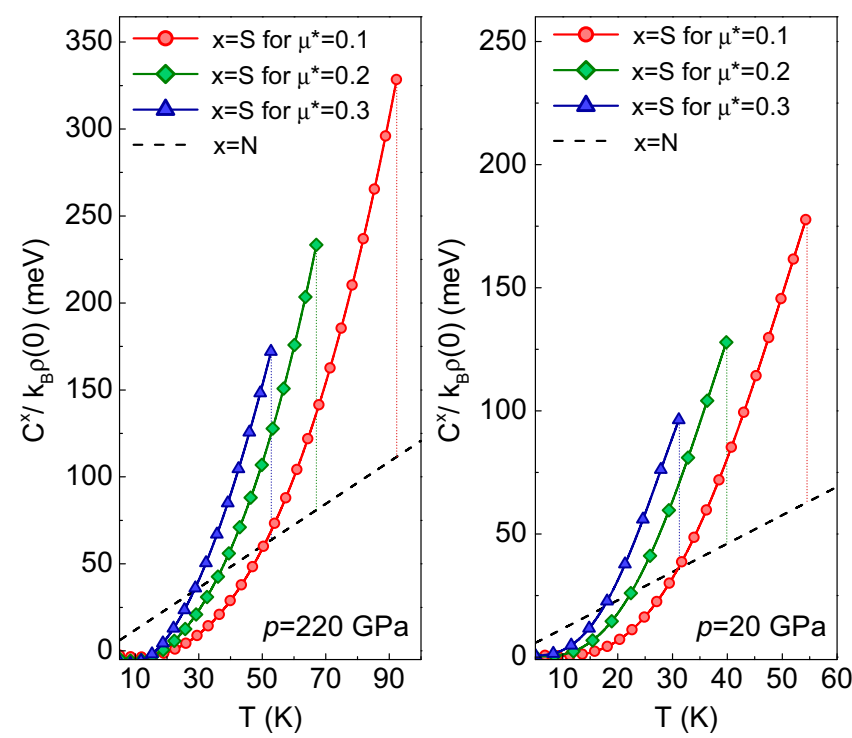

Fig. 4. The dependence of the specific heat in the superconducting $(x=S)$ and the normal $(x=N)$ state on the temperature for selected values of the Coulomb pseudopotential. The vertical dotted lines indicate the position of the specific heat jump at $T_{C}$.

theory, the values of dimensionless ratios, determined in this paper, differ significantly from the prediction of BCS theory. In particular, in Figure 5, we can see the dimensionless ratios as a function of the strong-coupling index $T_{C} / \omega_{\text {ln }}$ for $\mathrm{GeH}_{4}$ at 20 and $220 \mathrm{GPa}$ in comparison with experimental results reported for other conventional superconductors [29]. The solid lines correspond to the analytical formulas proposed in papers $[35,36]$

$$
\begin{gathered}
\frac{2 \Delta(0)}{k_{B} T_{C}}=3.53\left[1+12.5\left(\frac{k_{B} T_{C}}{\omega_{\ln }}\right)^{2} \ln \left(\frac{\omega_{\ln }}{2 k_{B} T_{C}}\right)\right] \\
\frac{\Delta C\left(T_{C}\right)}{C^{N}\left(T_{C}\right)}=1.43\left[1+53\left(\frac{k_{B} T_{C}}{\omega_{\ln }}\right)^{2} \ln \left(\frac{\omega_{\ln }}{3 k_{B} T_{C}}\right)\right]
\end{gathered}
$$

and

$$
\frac{T_{C} C^{N}\left(T_{C}\right)}{H_{C}^{2}(0)}=0.168\left[1-12.2\left(\frac{k_{B} T_{C}}{\omega_{\ln }}\right)^{2} \ln \left(\frac{\omega_{\ln }}{3 k_{B} T_{C}}\right)\right],
$$

where, the average phonon frequency $\left(\omega_{\ln }\right)$ is defined as:

$$
\omega_{\ln } \equiv \exp \left[\frac{2}{\lambda} \int_{0}^{+\infty} d \Omega \frac{\alpha^{2} F(\Omega)}{\Omega} \ln (\Omega)\right] .
$$

On the basis of above formulas it can be concluded that in the weak coupling BCS limit $T_{C} / \omega_{\mathrm{ln}} \rightarrow 0$ while in the case of $\mathrm{GeH}_{4}$ at $220 \mathrm{GPa}$ we have $T_{C} / \omega_{\text {ln }} \in\langle 0.11,0.06\rangle$.

It is clearly visible in Figure 5 that although the obtained results differ significantly from the prediction of the classic BCS theory, they are close to the general trend 


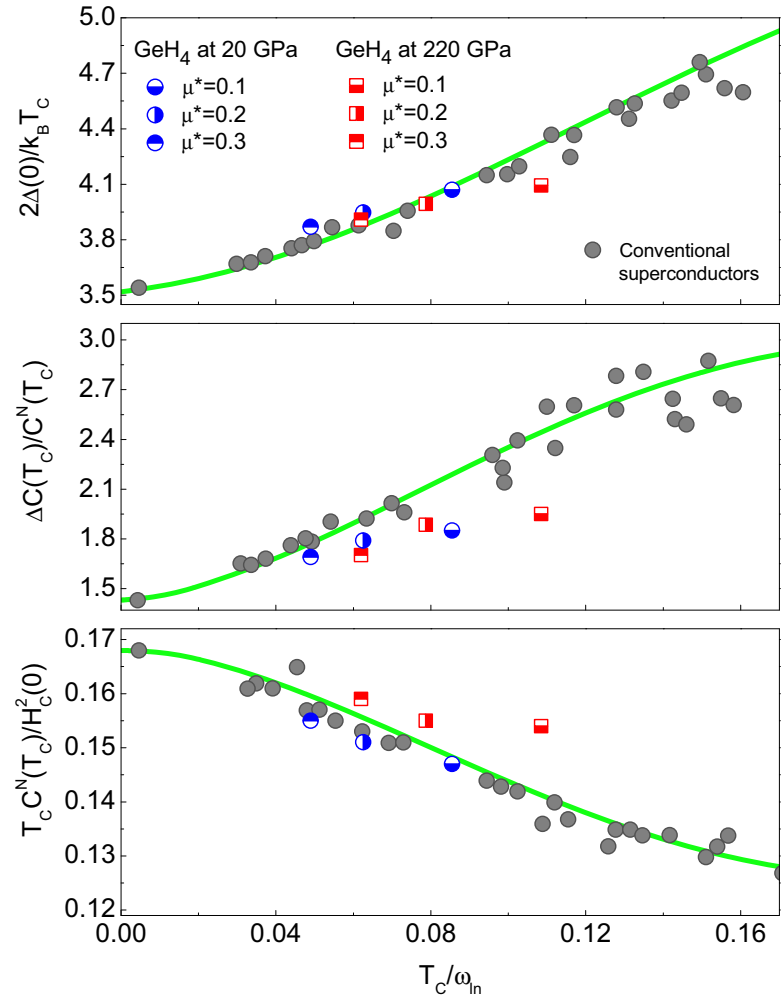

Fig. 5. The dimensionless ratios as a function of $T_{C} / \omega_{l n}$. The results for the selected conventional superconductors (full circles) are taken from paper [29]. The lines are obtained on the basic of papers $[35,36]$.

determined by the conventional superconductors. Significant derogations were observed only in the case of $\mathrm{GeH}_{4}$ at $220 \mathrm{GPa}$ for $\mu^{\star}=0.1$.

\section{Conclusions}

To summarize, we conducted the systematic study in order to describe the thermodynamic properties of $\mathrm{GeH}_{4}$ in the superconducting state (under the pressure of $220 \mathrm{GPa}$ ). In particular, the superconducting critical temperature, energy gap, free energy difference between the superconducting and normal state, thermodynamic critical field and the specific heat were determined for a wide range of Coulomb pseudopotential: $\mu^{\star} \in\langle 0.1,0.3\rangle$. It was stated, that investigated system is characterized by high critical temperature reaching a maximal value up to $92 \mathrm{~K}$ for $\mu^{\star}=0.1$.

In addition, our calculations suggest that the superconducting phase in GeH4 at $220 \mathrm{GPa}$ is thermodynamically more stable than in $\mathrm{GeH}_{4}$ at $20 \mathrm{GPa}$. Detailed comparison shows also that, due to the strong-coupling and retardation effects, both systems take non-BCS values of dimensionless ratios $2 \Delta(0) / k_{B} T_{C}, \Delta C\left(T_{C}\right) / C^{N}\left(T_{C}\right)$ and $T_{C} C^{N}\left(T_{C}\right) / H_{C}^{2}(0)$ but the obtained results agree with general trend appointed by conventional superconductors. Future experimental explorations on the superconductivity of this high-pressure system are highly desirable.

\section{Author contribution statement}

R. Szczesśniak participated in the design of this study, in writing the code for numerical calculations and in writing the final version of the manuscript. A.P. Durajski coordinated this study, carried out numerical calculations, drafted the manuscript, and participated in writing the final version of the manuscript. All authors read and approved the final manuscript.

All numerical calculations have been based on the Eliashberg function sent to us by Prof. Yanming Ma and Ph.D. Guoying Gao to whom we are very thankful.

\section{References}

1. N.W. Ashcroft, Phys. Rev. Lett. 92, 187002 (2004)

2. N.W. Ashcroft, Phys. Rev. Lett. 21, 1748 (1968)

3. V.V. Struzhkin, Physica C 514, 77 (2015)

4. M.I. Eremets, I.A. Trojan, S.A. Medvedev, J.S. Tse, Y. Yao, Science 319, 1506 (2008)

5. A. Drozdov, M.I. Eremets, I.A. Troyan, V. Ksenofontov, S.I. Shylin, Nature 525, 73 (2015)

6. A. Drozdov, M.I. Eremets, I.A. Troyan, arXiv: 1508.06224 (2015)

7. D.Y. Kim, R.H. Scheicher, C.J. Pickard, R.J. Needs, R. Ahuja, Phys. Rev. Lett. 107, 117002 (2011)

8. D. Szczęśniak, T.P. Zemła, Supercond. Sci. Technol. 28, $085018(2015)$

9. Y. Li, J. Hao, H. Liu, Y. Li, Y. Ma, J. Chem. Phys. 140, $174712(2014)$

10. G. Gao, H. Wang, A. Bergara, Y. Li, G. Liu, Y. Ma, Phys. Rev. B 84, 064118 (2011)

11. R. Szczȩśniak, A.P. Durajski, Supercond. Sci. Technol. 27, $015003(2014)$

12. D.Y. Kim, R.H. Scheicher, H.K. Mao, T.W. Kang, R. Ahuja, Proc. Natl. Acad. Sci. USA 107, 2793 (2010)

13. A.P. Durajski, R. Szczȩśniak, Supercond. Sci. Technol. 27, 115012 (2014)

14. G. Gao et al., Proc. Natl. Acad. Sci. U.S.A. 107, 1317 (2010)

15. G. Gao, A.R. Oganov, A. Bergara, M. Martinez-Canales, T. Cui, T. Iitaka, Y. Ma, G. Zou, Phys. Rev. Lett. 101, $107002(2008)$

16. C. Zhang, X.J. Chen, Y.L. Li, V. Struzhkin, R. Hemley, H.K. Mao, R.Q. Zhang, H.Q. Lin, J. Supercond. Nov. Magn. 23, 717 (2010)

17. G. Gao, R. Hoffmann, N.W. Ashcroft, H. Liu, A. Bergara, Y. Ma, Phys. Rev. B 88, 184104 (2013)

18. A.P. Durajski, Eur. Phys. J. B 87, 210 (2014)

19. H. Wang, J.S. Tse, K. Tanaka, T. Iitaka, Y. Ma, Proc. Natl. Acad. Sci. USA 109, 6463 (2012)

20. X. Feng, J. Zhang, G. Gao, H. Liu, H. Wang, RSC Adv. 5, $59292(2015)$

21. J.M. McMahon, M.A. Morales, C. Pierleoni, D.M. Ceperley, Rev. Mod. Phys. 84, 1607 (2012)

22. L. Sun, A.L. Ruoff, C.S. Zha, G. Stupian, J. Phys.: Condens. Matter 18, 8573 (2006)

23. X.J. Chen, V.V. Struzhkin, Y. Song, A.F. Goncharov, M. Ahart, Z. Liu, H.K. Mao, R.J. Hemley, Proc. Natl. Acad. Sci. USA 105, 20 (2008) 
24. G. Gao et al., Proc. Natl. Acad. Sci. USA 107, 1317 (2010)

25. M. Martinez-Canales, A.R. Oganov, Y. Ma, Y. Yan, A.O. Lyakhov, A. Bergara, Phys. Rev. Lett. 102, 087005 (2009)

26. Z. Li, W. Yu, C. Jin, Solid State Commun. 143, 353 (2007)

27. R. Szczȩśniak, A.P. Durajski, D. Szczȩśniak, Solid State Commun. 165, 39 (2013)

28. G.M. Eliashberg, Sov. Phys. J. Exp. Theor. Phys. 11, 696 (1960)

29. J. Carbotte, Rev. Mod. Phys. 62, 1027 (1990)

30. P. Giannozzi et al., J. Phys.: Condens. Matter 21, 395502 (2009)

31. F. Marsiglio, M. Schossmann, J.P. Carbotte, Phys. Rev. B 37, 4965 (1988)

32. J. Bardeen, M. Stephen, Phys. Rev. 136, A1485 (1964)
33. J. Bardeen, L.N. Cooper, J.R. Schrieffer, Phys. Rev. 106, $162(1957)$

34. J. Bardeen, L.N. Cooper, J.R. Schrieffer, Phys. Rev. 108, 1175 (1957)

35. B. Mitrović, H.G. Zarate, J.P. Carbotte, Phys. Rev. B 29, $184(1984)$

36. F. Marsiglio, J.P. Carbotte, Phys. Rev. B 33, 6141 (1986)

Open Access This is an open access article distributed under the terms of the Creative Commons Attribution License (http://creativecommons.org/licenses/by/4.0), which permits unrestricted use, distribution, and reproduction in any medium, provided the original work is properly cited. 